\title{
Quaderni
}

QUADERNI Communication, technologies, pouvoir

68 | Hiver 2008-2009

Militantisme médical et fabrique des politiques de santé

\section{Quand les acquis d'un mouvement social créent les conditions d'un militantisme médical : l'exemple des sourds}

Sophie Dalle-Nazébi et Nathalie Lachance

\section{(2) OpenEdition}

\section{Journals}

Édition électronique

URL : http://journals.openedition.org/quaderni/281

DOI : 10.4000/quaderni.281

ISSN : 2105-2956

Éditeur

Les éditions de la Maison des sciences de l'Homme

Édition imprimée

Date de publication : 5 janvier 2009

Pagination : 83-85

\section{Référence électronique}

Sophie Dalle-Nazébi et Nathalie Lachance, "Quand les acquis d'un mouvement social créent les

conditions d'un militantisme médical : l'exemple des sourds », Quaderni [En ligne], 68 | Hiver

2008-2009, mis en ligne le 05 janvier 2012, consulté le 20 avril 2019. URL : http://

journals.openedition.org/quaderni/281; DOI : 10.4000/quaderni.281 
quand les acquis d'un mouvement social créent les conditions d'un militantisme médical : l'exemple des sourds

\author{
Sophie \\ Dalle-Nazébi \\ sociologue \\ LISST-CERS, Toulouse 2 \\ Nathalie \\ Lachance
}

anthropologue, professeure associée Département de Service Social Université de Sherbrooke Canada
Plusieurs débats traversent les sciences de la vie concernant la manière de penser la surdité et, audelà, les rapports entre nature et culture. $\mathrm{Si}$, pour la plupart d'entre nous, la surdité se résume à une déficience auditive, des sourds ont, pour leur part, une représentation culturelle endogène - propre et spécifique - d'eux-mêmes. Mais toutes les personnes atteintes de surdité ne se disent ni ne sont reconnues comme « Sourdes ». Comme en attestent notre terrain en France et au Québec depuis quinze ans, c'est par l'insertion dans des collectifs de sourds que s'acquièrent les manières de faire, de penser et d'être propres à ce groupe. Se présenter comme « un Sourd »-plutôt que comme une personne atteinte d'une déficience auditive - c'est donc faire référence à un groupe d'individus partageant une histoire, une langue gestuelle et une culture. Il n'y aurait pas ici de corps déficients à réparer, ni de drame dans la naissance d'enfants sourds. Les sourds, ainsi définis, refusent à tout autre groupe ou acteur la possibilité de définir à leur place ce qu'ils sont, et ce que signifie « être sourd» : « Nous, les sourds, en avons assez d'une certaine forme de paternalisme et de mise en tutelle qui ne laissent pratiquement aucune issue à ceux qui en subissent la contrainte $»^{1}$. Leurs revendications (rassemblées à partir d'entretiens en langue des signes et d'archives de presse) relèvent ainsi d'abord d'un registre émancipatoire : ils revendiquent un savoir spécifique, tiré de leurs expériences, sur leurs problèmes et sur la manière de les résoudre; ils réclament la prise en compte de leurs difficultés par les autorités; ils refusent d'être les objets passifs des analyses pour en devenir, à l'inverse, les principaux acteurs².

Les sourds contestent aux scientifiques «spé- 
cialistes de la surdité », la pertinence de leurs études et leur légitimité. Ils font valoir, à partir des mobilisations des années 1980, leur propre expertise sur la surdité, que ce soit la leur ou celle d'enfants avec qui ils n'ont souvent aucun lien biologique (la majorité des sourds ayant des parents qui entendent): "plus que les entendants même bourrés de titres universitaires, pour l'avoir vécu avec nos tripes nous savons ce que représente pour nous notre surdité ainsi que les rapports que les personnes de l'institution, chargées de notre "bien-être "entretiennent avec nous $»^{3}$. À travers ces mobilisations qui ne portent pas sur la santé, mais sur l'éducation, la formation et l'emploi, les sourds ont acquis un statut d'interlocuteurs auprès des pouvoirs publics en raison d'une expérience que d'autres n'ont pas : des compétences linguistiques particulières qu'ils peuvent transmettre aux plus jeunes et la possibilité d'incarner un modèle d'adultes sourds. Ces mobilisations sont alors décisives dans la structuration récente d'un militantisme médical en faveur de l'accès aux services de soin en langues des signes, et contre la pose d'implant cochléaire par chirurgie sur de jeunes enfants sourds et l'essor du dépistage génétique précoce de la surdité.

Beaucoup de sourds voient dans l'appareillage et la chirurgie de l'oreille un acharnement thérapeutique et un déni de leurs compétences. Au Québec, ils organisent au milieu des années 1990 des rassemblements devant le Parlement et des marches sur la place publique pour protester contre les implantations cochléaires chez les enfants et exiger un moratoire. En France, ils s'inspirent des acteurs prônant d'autres rapports entre malades et médecins, comme Act Up. Les sourds découvrent en effet simultanément les risques du sida et de l'implant, tout deux associés - à leurs yeux -à la mort. Ce militantisme assure la présence de sourds dans le milieu médical. Mais en prenant part aux groupes de travail sur les usages de l'implant, ceux-ci cautionnent - par leur simple présence - une pratique qu'ils récusent. Il en est de même au Québec où des sourds s'investissent, en tant qu'éducateurs spécialisés ou professeurs de Langue des Signes Québécoise, dans des centres de réadaptation où l'implant n'est plus contesté. Si les sourds s'interrogent sur les positions à adopter devant cet état de fait, les récentes mobilisations sur le dépistage génétique montrent leur apprentissage des procédures juridiques en matière d'éthique. Celui-ci s'est fait à travers l'organisation de services de soins en langues des signes, supposant l'initiation de médecins aux langues des signes et les sourds aux métiers de soignants. L'histoire des rencontres entre ces acteurs, et celle des relations entre communautés sourdes, expliquent les formes que prennent ces services comme ces mobilisations dans différents pays. Ainsi, les déclarations politiques faites en Belgique - où se pratique la Langue des Signes Française articulant dépistage et implant, expliquent que ces deux débats soient désormais associés en France - contrairement au Québec -, et que les sourds y aient interpellé le Comité Consultatif National d'Éthique sur ces questions.

La recherche engagée ici dans une perspective comparative concerne ainsi les formes et les conditions d'existence de ces actions collectives. Ces controverses sont en effet le fruit des avancées médicales mais aussi celui de mouvements sociaux plus anciens de sourds qui ont 
considérablement transformé, en trente ans, les pratiques administratives et politiques de prise en charge des personnes sourdes. Ces mouvements eux-mêmes ne peuvent être compris en dehors d'une approche historique et anthropologique des collectifs de sourds. Il est donc nécessaire d'articuler les outils et références théoriques des recherches sur les sciences et les techniques, les formes d'actions collectives et les phénomènes culturels.
$\mathrm{N} \cdot \mathrm{O} \cdot \mathrm{T} \cdot \mathrm{E} \cdot \mathrm{S}$

1. Roland Major, « Les États Généraux de la déficience auditive au Québec dans l'optique d'un sourd», in Chronique du Club abbé de l'Épée, n 8.4., 1973, p. 4.

2. Vololonna Rabeharisoa, «Forms of Involvement of Patient Organisations into Research. An Overview of Different Models », in Stage Opening Conference, Copenhagen, 10-11 janvier, 2002.

3. Anonyme, "Les sourds de Poitiers ont écrit», in Journal de Liaison $2 L P E, \mathrm{n}^{\circ} 3 \& 4$, 1983, p. 35. 
